\title{
A note on auditory neurons having periodic response functions to time-delayed, binaural stimuli*
}

\author{
DENNIS McFADDEN \\ University of Texas, Austin, Texas 78712
}

\begin{abstract}
So-called cyclic or periodic response functions have been reported for single neurons responding to interaural differences in time of arrival. These data have apparently been misinterpreted by some, for in real-world situations these cells would not be the source of ambiguous localization information for higher auditory centers.
\end{abstract}

Apparently there is confusion, at least among some psychoacousticians, about certain recent neurophysiological findings on the processing of binaural inputs by single auditory neurons. While it is unlikely that the neurophysiologists are themselves confused about the facts, they have failed to make explicit the explanation of one of their commonly reported outcomes, and this is apparently the reason for the misunderstanding among their nonphysiological readers.

Sensitivity to interaural time differences has been studied by many investigators working with neurons at several different levels in the auditory nervous system. In a typical experiment, binaural stimuli (commonly clicks) are presented, one to each ear, with an interaural time difference that is varied across presentations. The data are typically presented in a figure having an abscissa of interaural time difference $(\Delta t)$ and an ordinate of firing rate or "probability" of firing. In such a coordinate system, the data take several forms, with perhaps the most common being a marked increase or decrease in firing rate, or firing probability, when the temporal separation between the two clicks is within a certain critical range. For many cells, however, there is not a single range of values of $\Delta t$ that produces the characteristic change in firing, but rather several different ranges are evident, each having about the same effect on the response rate (for example, see Brugge, Dubrovsky, Aitkin, \& Anderson, 1969; Goldberg \& Brown, 1969; Hall, 1965; Rose, Gross, Geisler, \& Hind, 1966). In fact, these data often take the form of a cyclic or periodic function, and it is these cyclic functions that have apparently been misunderstood. Some have interpreted such data to mean that these cells are "tuned" to multiple values of $\Delta t$, a most perplexing. conclusion indeed if these cells were involved in auditory localization, for it would imply that they could be activated by sound sources located at a number of different points off the median plane. Obviously, if this were true, then activation of a particular cell would not uniquely signal a single angular position for the sound

*The publication of this note was supported by a grant from the Public Health Service, National Institute of Neurological Diseases and Stroke. source. However, the likelihood is that this interpretation is in error and that these cells are not a source of ambiguous localization information for higher neural centers.

Two important facts are being overlooked by those who have interpreted the cyclic data in this way. The first fact is that a single click delivered to one ear does not produce a single firing or a single burst of firing in the primary auditory neurons. Rather, each neuron responds with a succession of spikes which are separated by integral multiples of the period of the characteristic frequency of that neuron. Said differently, the single acoustic event produces "ringing" at each location along the basilar membrane, and this ringing manifests itself neurally as a succession of more or less evenly spaced firings. A click of duration $100 \mu \mathrm{sec}$ can produce such "phased" neural activity for as long as $8-10 \mathrm{msec}$ (see Kiang, 1965). Given this fact, it is easy to see how one could produce cyclic enhancements or depressions in the firing rate of a higher-order cell sensitive to a particular interaural time difference. When the first spike potential from the leading ear and the first from the lagging ear are in the proper temporal arrangement, the cell will demonstrate its characteristic change (increase or decrease in rate). But it will also show this same response when the click to the lagging ear is delayed by an amount such that the second spike from the leading ear and the first from the lagging ear are properly aligned temporally. Obviously, the third spike from the leading ear and the first spike from the lagging ear could also be properly aligned by further delaying the click to the lagging ear. In general, once the $\mathrm{E}$ has established the particular value of $\Delta t$ to which a cell is tuned, then any interaural delay equal to the sum of that value of $\Delta t$ plus integral multiples of the period of the cell's characteristic frequency ought to lead to the same effect on the cell. The range of values of time delay for which this will occur will depend upon how long the system rings, which in turn depends upon such factors as the intensity of each of the two clicks and the characteristic frequency (i.e., the distance from the stapes) of the primary neurons involved.

Of course, the general form of this explanation also holds for cyclic response functions obtained with 
continuous waveforms such as sinusoids. The primary difference is that it is the stimulus itself and not the ringing of the peripheral auditory system that is the source of the succession of temporally discrete spike potentials; that is, there is phase-locked responding to continuous sinusoids of about $4,000-5,000 \mathrm{~Hz}$ and below. Thus, the cyclic response functions obtained with tonal stimuli ought to have "periods" corresponding to integral multiples of the period of the particular sinusoid being used, not to integral multiples of the period of the characteristic frequency of the cell (for confirmation, see Fig. 11 of Brugge, Dubrovsky, Aitkin, \& Anderson, 1969; Fig. 1 of Brugge, Anderson, \& Aitkin, 1970; Fig. 3A of Rose, Gross, Geisler, \& Hind, 1966).

The second fact being overlooked by those who have misinterpreted cyclic response functions is that the cyclic form of these data is usually obvious only when values of $\Delta t$ much greater than one headwidth of the animal are used. In the real world, of course, it is impossible for a single wavefront to acquire such large interaural differences in arrival time (see McFadden, 1973). Thus, it is clear that in real-world situations, these cells will always be operating over the segment of their cyclic response function contained within plus-and-minus one headwidth of zero interaural delay-and their activation will therefore be singularly unambiguous as to the angular position of the sound source.

\section{REFERENCES}

Brugge, J. F., Dubrovsky, N. A., Aitkin, L. M., \& Anderson, D. J. Sensitivity of single neurons in auditory cortex of cat to binaural tonal stimulation; effects of varying interaural time and intensity. Journal of Neurophysiology, 1969, 32, 1005-1024.

Brugge, J. F., Anderson, D. J., \& Aitkin, L. M. Responses of neurons in the dorsal nucleus of the lateral lemniscus of cat to binaural tonal stimulation. Journal of Neurophysiology, 1970, 43, 441-458.

Goldberg, J. M., \& Brown, P. B. Response of binaural neurons of dog superior olivary complex to dichotic tonal stimuli: Some physiological mechanisms of sound localization. Journal of Neurophysiology, 1969, 32, 613-636.

Hall, J. L., II. Binaural interaction in the accessory superior-olivary nucleus of the cat. Journal of the Acoustical Society of America, 1965, 37, 814-823.

Kiang, N. Y.S. Discharge patterns of single fibers in the cat's auditory nerve. Cambridge, Massachusetts: M.I.T. Press, 1965.

McFadden, D. Precedence effects and auditory cells with long characteristic delays. Journal of the Acoustical Society of America, 1973, in press.

Rose, J. E., Gross, N. B., Geisler, C. D., \& Hind, J. E. Some neural mechanisms in the inferior colliculus of the cat which may be relevant to localization of a sound source. Journal of Neurophysiology, 1966, 29, 288-314.

(Received for publication April 5, 1973; accepted April 13, 1973.) 